\title{
Vegetation Database East Africa
}

\author{
Andreas Hemp
}

\begin{abstract}
Since 1991, nearly 1800 plots in all vegetation formations of East Africa (Ethiopia, Kenya and Tanzania with focus on the Kilimanjaro) were established using the method of Braun-Blanquet (1964). Inside the highly complex und structered montane forests the study included for the first time in tropical regions all vegetation layers (herbs, shrubs, trees, lianas and epiphytes). On the mountains of Tanzania these plots are equipped with data loggers recording climatic parameters. Aim of the database: classification and vegetation map of East Africa (a detailed map of Kilimanjaro is already prepared); reconstruction of vegetation and climate history of East Africa (in collaboration with studies of flightless Saltatoria and palaeobotany); analysis of diversity patterns along altitudinal gradients; providing baseline data for the DFG research unit KiLi (FOR 1246). This report describes the available content in the vegetation-plot database of the Vegetation Database East Africa (GIVD ID AF-00-004).
\end{abstract}

Keywords: altitudinal gradient; Kilimanjaro; tropical montane forest; vegetation history East Africa.

\section{Vegetation Database East Africa}

Scope: Vegetation analysis in Ethiopia, Kenya and Tanzania. Diversity patterns along altitudinal gradients, baseline data for the new DFG research unit KiLi on Kilimanjaro, as well as for a palaeobotanical DFG project in Tanzania.

Status: ongoing capture Period: $1991-2012$

Database manager(s): Andreas Hemp (andreas.hemp@uni-bayreuth.de)

Owner: Andreas Hemp

Web address: [NA]

Availability: according to a specific agreement

Database format(s): [NA]

Publication: [NA]

Plot type(s): normal plots

Non-overlapping plots: 1,850

Total plot observations: 1,850

Online upload: no

Online search: no

Export format(s): [NA]

Plot-size range: 5-1,000 $\mathrm{m}^{2}$

Countries: ET: $2.0 \%$; KE: 5.0\%; TZ: $93.0 \%$

Forest: 35\% — Non-forest: aquatic: $1 \%$; semi-aquatic: $5 \%$; arctic-alpine: $8 \%$; natural: $26 \%$; semi-natural: $8 \%$; anthropogenic: $16 \%$

Guilds: all vascular plants: $100 \%$

Environmental data: altitude: $100 \%$; slope aspect: $100 \%$; slope inclination: $100 \%$; soil depth: $100 \%$; surface cover other than plants (open soil, litter, bare rock etc.): $5 \%$; soil pH: $20 \%$; other soil attributes: $100 \%$

Performance measure(s): cover: $100 \%$

Geographic localisation: GPS coordinates (precision $25 \mathrm{~m}$ or less): $90 \%$

Sampling periods: 1990-1999: 42.0\%; 2000-2009: 55.0\%; 2010-2019: 3.0\%

Information as of 2012-07-12; further details and future updates available from http://www.givd.info/ID/AF-00-004

Andreas Hemp (andreas.hemp@uni-bayreuth.de)

Department of Plant Systematics, BAYCEER, University of Bayreuth, Dr. Hans-Frisch-Str. 1-3, 95448 Bayreuth, GERMANY 